\title{
Anomalous microwave emission from spinning nano-diamonds around stars
}

\author{
Authors: J.S. Greaves ${ }^{1 *}$, A.M.M. Scaife ${ }^{2}$, D.T. Frayer ${ }^{3}$, D.A. Green ${ }^{4}$, B.S. Mason ${ }^{5}$, A.M.S. Smith ${ }^{6}$
}

Affiliations:

${ }^{1}$ School of Physics \& Astronomy, Cardiff University, 4 The Parade, Cardiff CF24 3AA, UK.

${ }^{2}$ Jodrell Bank Centre for Astrophysics, AlanTuring Building, Oxford Road, Manchester M13 9PL, UK.

${ }^{3}$ Green Bank Observatory, PO Box 2, Green Bank, WV 24944, USA.

${ }^{4}$ Astrophysics Group, Cavendish Laboratory, J.J. Thomson Avenue, Cambridge CB3 0HE, UK.

${ }^{5}$ National Radio Astronomy Observatory, 520 Edgemont Road, Charlottesville, VA 22903, USA.

${ }^{6}$ DLR, Institut für Planetenforschung, Rutherfordstraße 2, 12489 Berlin, Germany.

*Correspondence and material request to: GreavesJ1@cardiff.ac.uk.

Several interstellar environments produce 'anomalous microwave emission', with brightness-peaks at tens-of-gigahertz frequencies ${ }^{1}$. The emission's origins are uncertain rapidly-spinning nano-particles could emit electric-dipole radiation ${ }^{2}$, but polycyclic aromatic hydrocarbons proposed as the carrier are now found not to correlate with Galactic signals ${ }^{3,4}$. The difficulty is to identify co-spatial sources over long lines of sight. Here we identify anomalous microwave emission in three proto-planetary discs. These are the only known systems that host hydrogenated nano-diamonds ${ }^{5}$ in contrast to very common detection of polycyclic aromatic hydrocarbons ${ }^{6}$. Spectroscopy locates the nanodiamonds close to the host-stars, at physically-constrained temperatures ${ }^{7}$. Developing disc models $^{8}$, we reproduce the emission with diamonds 0.75-1.1 nanometres in radius, holding $\leq 1-2 \%$ of the carbon budget. The microwave-emission:stellar-luminosity ratios are approximately constant, allowing nano-diamonds to be ubiquitous but emitting below detection thresholds in many star-systems. This can unify the findings with similar-sized diamonds found within solar system meteorites ${ }^{9}$. As nano-diamond spectral absorption is seen in interstellar sightlines ${ }^{10}$, these particles are also a candidate for generating galaxyscale $^{3}$ anomalous microwave emission.

Here we analyse discs around luminous stars, mainly Herbig A-type emission-line (HAe) objects, rather than 'classical T Tauri' stars (CTTS) like the young Sun (where the discs evolve somewhat differently ${ }^{11,12}$ ). Disc masses are characterised using thermal radiation from dust grains, and superposed spectral features indicate types of small particles, down to molecular sizes, e.g. polycyclic aromatic hydrocarbons (PAHs). One prominent PAH feature is at $3.3 \mu \mathrm{m}$, and this infrared band has also revealed ${ }^{13}$ a few sources with $3.43,3.53 \mu \mathrm{m}$ peaks. These features are identified $^{5}$ with hydrogenated nano-diamonds ${ }^{14}$, of special interest as connecting to solar system nano-diamonds in meteorites ${ }^{9}$. The diamonds may form under conditions of high pressure, shocks or vapour deposition ${ }^{15}$, either internal or external to the proto-solar and extrasolar discs. We discuss the only three extrasolar discs found to host nano-diamonds, after systematic searches ${ }^{5,16}$ of over 80 HAe stars. Many HAes ${ }^{6}$ and some CTTS ${ }^{17}$ exhibit PAHs, while diamonds could be rather ubiquitous but only rarely sufficiently excited to produce infrared features ${ }^{5}$. 
Nano-particle carriers of anomalous microwave emission (AME) have been extensively sought, and imperfect correlation of $\mathrm{PAH}$ and $\mathrm{AME}$ sightlines ${ }^{4}$ is broadening investigations to hydrocarbon-carriers of diffuse interstellar absorption bands ${ }^{18}$ (DIBs) and silicate/iron nanograins $^{4,19}$. While sophisticated models exist, observations are hampered by not knowing if spectral and AME sources are co-spatial. Here we address this by identifying AME in circumstellar discs, at specific locations. Notably, AME is found only in discs hosting hydrogenated nano-diamonds, whose surface $\mathrm{C}-\mathrm{H}$ bonds can provide the required electric dipole moments.

The AME was discovered from distinctive peaked microwave spectra, dissimilar to power-law flux-distributions from free-free electron-transitions in winds/jets, gyrosynchrotron emission from stellar-surface spots, and thermal emission from dust. Use of several radio bands quasisimultaneously also helped to identify AME, especially as some small-scale processes are timevariable. Two independent radio surveys were made, covering 9 HAe systems at the Australian Compact Telescope Array (ATCA), and 5 systems with primaries ${ }^{11}>1 \mathrm{M}_{\text {sun }}$ observed at the $100 \mathrm{~m}$ Robert C. Byrd Green Bank Telescope (GBT). The ATCA interferometer filters out any extended structure, with beams of $\sim 35$ down to $\sim 3$ arcseconds at 5.5-97 GHz isolating the discs; some noncontemporaneous archival data were also processed to fill out frequency coverage. The GBT scans an $\sim 25$ arcsec beam at $26-40 \mathrm{GHz}$ to filter out extended emission, producing photometry in four sub-bands simultaneously. Follow-up measurements were made with the Arcminute Microkelvin Imager (AMI) at $16 \mathrm{GHz}$ and GBT at $72 \mathrm{GHz}$; all new data are shown in Supplementary Figures 1-3.

Contributing signals from dust and winds were subtracted first, and then residuals for three AME-candidate discs were fitted. The subtraction procedure sought to minimise residuals over all wavelengths, within the constraint that these should not be negative (within the errorbars, which fold in noise, calibration, and temporal variability). The three sources remaining are thus those where AME is essential in order to reproduce the microwave emission. Full details of procedures are given in the Methods and in Supplementary Figures 1-3, Tables 1-3.

The AME flux-profiles are shown in Figs 1-3. In the V892 Tau system, the AME peaks at a frequency around $25 \mathrm{GHz}$, and has a maximum amplitude of $\approx 1 \mathrm{mJy}$ (over half the total signal, Fig. S3). The GBT residuals are independently confirmed from ATCA data ${ }^{20}$. HD 97048 has a similar AME amplitude, inferred from our two ATCA points, while the peak frequency is shifted slightly lower, to $\approx 20 \mathrm{GHz}$. In the luminous MWC 297 system, the peak frequency lies at $~ 50$ $\mathrm{GHz}$ (between our ATCA and GBT bands), and the AME amplitude is much higher, at $30 \mathrm{mJy}$.

Model AME spectra were constructed from a disc formulism ${ }^{8}$ for spinning nano-particles. For a sphere large enough to behave classically, the power radiated is

$P(a, \omega)=(4 / 9) d^{2}(a) \omega^{4} / \mathrm{c}^{3}$

for particle radius $a$, spin frequency $\omega$ and electric dipole moment $d$. Prior work ${ }^{2,8}$ set $d=N^{1 / 2} \mathrm{~d}_{0}$ for $\mathrm{N}$ randomly-oriented surface dipoles, with e.g. a C-H bond having $\mathrm{d}_{0} \approx 0.4$ Debye. However, in symmetric diamondoids these dipoles would self-cancel, so here $d$ is assumed dominated by ionised and asymmetric forms $\mathrm{s}^{21,22}$ with $d \sim 0.5-5.7$ Debye. In Table 1 , we fit with a mid-range $d=$ 1.5 Debye, and subsequently scale radiated power $P$ by $d^{2}$ (Eq. 1 ).

For a Boltzmann distribution of spin rates given by

$f(\omega)=4 \pi(3 / 2 \pi)^{3 / 2}\left(\omega^{2} /<\omega^{2}>^{3 / 2}\right) \exp \left(-3 / 2 \omega^{2} /<\omega^{2}>\right)$, 
the expectation value of the emission frequency (in radians/sec) for temperature $T$ and moment of inertia $I$ is

$<\omega^{2}>^{1 / 2}=\left(3 \mathrm{k}_{\mathrm{B}} T / I\right)^{1 / 2}$,

varying as $T^{1 / 2} \rho^{-1 / 2} a^{-5 / 2}$; mass-density $\rho \approx 2.5 \mathrm{~g} / \mathrm{cm}^{3}$ is adopted around C-atoms spaced ${ }^{23}$ by 0.2 $\mathrm{nm}$. Model spheres were assumed, to approximate to diamond chunks within meteorites and to match prior work ${ }^{8}$, but noting that $d \neq 0$ actually requires asymmetry or charge. We fit for one characteristic $a$ rather than a distribution, as cage-structured diamonds have quantised sizes ${ }^{23}$.

The AME spectrum is then given by

$L(a)=n($ diamonds $) / n(\mathrm{H}) \int 2 \pi R \Sigma(R) / \mathrm{m}_{\mathrm{H}} \mathrm{d} R . P(a, \omega) .2 \pi f_{\omega}(\omega, R)$,

integrated over a disc's radial mass-surface-density $\Sigma(R)$. Two nano-diamond surface-profiles are unresolved ${ }^{24,25}$, with V892 Tau fits ${ }^{7}$ declining as an $R^{\sim-0.3}$ function (but subject to assumed particle size). Table 1 results are for a flat $\Sigma(R)$, while steeper profiles tested for V892 Tau yielded lower nano-diamond abundances.

Particle temperatures here assume local thermodynamic equilibrium, not strictly valid for sizes $<2 \mathrm{~nm}$ with transient heating ${ }^{26}$. Hydrogenated nano-diamonds are only stable ${ }^{7}$ at $\mathrm{T} \approx 800-1400 \mathrm{~K}$, so observed $R_{\text {inner }}$ (V892 Tau) or $R_{\text {outer }}$ (HD 97048, MWC 297) set the upper or lower temperature bound, with the other radius set in thermal equilibrium $\left(T \propto R^{-0.5}\right)$. The warm temperatures suggest nano-particles floating in the largely-atomic layers above and below the disc mid-plane.

The AME fits (Figs 1-3, Table 1) are functions of only two free parameters, nano-diamond size and abundance (against total carbon, with cosmic $\mathrm{C}: \mathrm{H}=4 \times 10^{-4}$ ). The model spheres are found to have similar radii, of $0.75-1.1 \mathrm{~nm}$, in all three systems. The different AME peak-frequencies have emerged naturally from the varying disc sizes and stellar luminosities, without requiring diverse nano-particles. These model spheres equate to $200-700 \mathrm{C}$-atoms, while for equal counts, the smallest tetrahedral equivalent would be a 6-layer pyramid ${ }^{23}, 1.8 \mathrm{~nm}$ on a side, and 'boxy' forms $^{21}$ would be longer. In the case of HD 97048, the nano-diamond spectra (Fig. S4) have a possible fit with a comparable 5-layer pyramid ${ }^{23}$. Similarly-scaled nano-diamonds within meteorites $^{9}$ are often around 2-3 nm across. One model uncertainty is the excitation temperature of the particles, potentially much lower than kinetic temperature in diffuse gas ${ }^{18}$, e.g. in high disc layers. We implicitly took $T$ as kinetic temperature, but at an extreme with particles excited to only $\sim 10 \mathrm{~K}$ (estimated for diffuse interstellar gas ${ }^{18}$ ), sizes would shrink from $0.75-1.1 \mathrm{~nm}$ to $\sim 0.3-0.45 \mathrm{~nm}$ (Eq. 3). This lower size-bound corresponds to the smallest possible nano-diamond species, adamantane $\left(\mathrm{C}_{10} \mathrm{H}_{16}\right)$, which may contribute ${ }^{23}$ to the infrared spectrum of HD 97048 .

The nano-diamond abundances would scale up to at most $1-2 \%$ of total carbon, for dipole moments reduced down to 0.4-0.5 Debye (single C-H bond or single charge). These abundances do not exceed 1-3\% diamond:C ratios estimated to reproduce interstellar $3.47 \mu \mathrm{m}$ nano-diamond bands, of similar absorbance ${ }^{21}$ to $3.43 / 3.53 \mu \mathrm{m}$ features. If total carbon-budgets locked up in small species are interstellar-like ${ }^{8}$, at $\sim 5 \%$, the nano-diamonds in the discs fit well inside this constraint.

Our results closely associate circumstellar AME with the presence of nano-diamonds. In total, 39 discs were surveyed with GBT/ATCA, with 14 having luminous host-stars (Supplementary Tables 1-3). AME was found only in the three discs with nano-diamond signatures, not generically among luminous-star discs (of which 85-100\% exhibit PAHs, Supplementary Figure 5) or towards CTTS. To estimate a false-alarm probability, we counted nano-diamond IR- 
detections within comprehensive HAe samples ${ }^{5,16}$, yielding $3 / 82$, a probability $\mathrm{P}_{\text {nano }}=0.037$ per star. The chance-probability in a 14-star sample of picking the three stars with AME and finding they have nano-diamonds, and also finding the remaining 11 stars do not, is $\mathrm{P}_{\text {chance }}=\mathrm{P}_{\text {nano }}{ }^{3}$ ( 1 $\left.\mathrm{P}_{\text {nano }}\right)^{11}$, or $0.003 \%$. This is robust against removing individual systems: e.g. CD-42 11721 is now known to be unusually distant, so a better estimate is $\mathrm{P}_{\text {nano }}{ }^{3}\left(1-\mathrm{P}_{\text {nano }}\right)^{10}$, i.e. $\mathrm{P}_{\text {chance }}=0.0035 \%$. Conservatively, we also checked biases towards discs with brighter dust or PAHs in our target selection, which could have eliminated some of the nano-diamond IR-sample. This could raise $\mathrm{P}_{\text {nano }}$ to $\approx 0.06$, and $\mathrm{P}_{\text {chance }}$ to $\approx 0.01 \%$, still very low.

Table 1. Data are listed for star and disc parameters, along with model results fitting the anomalous microwave emission. ML and Ex entries indicate maximum likelihood and expectation fits respectively. These tabulated solutions are for flat surface-density profiles, with disc-mass estimates based uniformly on dust fluxes measured near $110 \mathrm{GHz}$ (in the optically-thin regime); the abundances (diamond: carbon

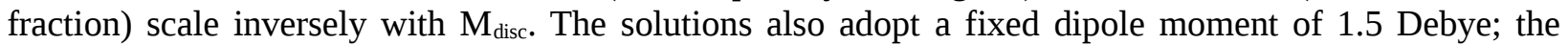
abundance scales as $d^{-2}$ (Eq. 1), with different diamond forms having $d \approx 0.4-5.7$ Debye.

\begin{tabular}{|l|l|l|l|}
\hline & V892 Tau & HD 97048 & MWC 297 \\
\hline$d$, star distance (parsecs) & $142 \pm 14$ & $158 \pm 16$ & $250 \pm 50$ \\
\hline$T_{\text {eff, }}$ effective temperature (kelvin) & 11,200 & 15,000 & 24,500 \\
\hline$L_{*}$ (solar luminosities) & $\sim 80(40-96)$ & $\sim 40(30-50)$ & $\sim 21,000(12,000-32,000)$ \\
\hline$M_{*}$ (solar masses) & $\sim 5.5(\sim 8$ AU binary) & 2.5 & $\sim 10$ \\
\hline$M_{\text {disc }}$ (solar masses) & 0.035 & 0.11 & 0.4 \\
\hline$R$ (diamonds) in model (AU) & $10-30$ & $5-15$ & $40-120$ \\
\hline$a$, size of model diamonds (nm) & $0.95(\mathrm{ML})$ & $1.05(\mathrm{ML})$ & $0.74(\mathrm{ML})$ \\
\hline diamond:carbon fraction (\%) & $0.952 \pm 0.015(\mathrm{Ex})$ & $1.07 \pm 0.05(\mathrm{Ex})$ & $0.75 \pm 0.03(\mathrm{Ex})$ \\
\hline$L_{\mathrm{AME}}$ (solar luminosities) & $0.13 \pm 0.02(\mathrm{Ex})$ & $\begin{array}{l}0.07(\mathrm{ML}) \\
0.08 \pm 0.03(\mathrm{Ex})\end{array}$ & $\begin{array}{l}0.18(\mathrm{ML}) \\
0.19 \pm 0.03(\mathrm{Ex})\end{array}$ \\
\hline
\end{tabular}

In proto-planetary discs, the carrier of AME is strongly indicated to be hydrogenated nanodiamonds. PAHs are less plausible as our sample has numerous PAH-hosting discs without AME. In particular, there are discs brighter in PAH features (Supplementary Figure 6) than V892 Tau and HD 97048, that do not show AME. Further, there is a divide in parameter space between the disc with nano-diamond IR-emission and AME, and systems without either phenomenon.

We hypothesize that more distant and/or lower-luminosity star-systems have less-detectable AME, but still could host nano-diamonds - this might link our findings with diamonds found around the Sun. The ratios of AME:stellar-luminosity are $\approx(1-3) \times 10^{-9}$ (constant within uncertainties, Table 1), even though the three stars span a range $\sim 500 \mathrm{in} \mathrm{L*}$. Hence detection of AME in our sample may be flux-limited; only AB Aur modestly challenges a constant $\mathrm{L}(\mathrm{AME}) / \mathrm{L} *$ scenario, at an upper limit of $\approx 0.3 \times 10^{-9}$. There is no corresponding simple relationship of nano-diamond IR-fluxes to host-star properties, after nearly four decades of 
study $^{5,13}$. However, the three detected systems are among the four hottest stars in our sample, with only CD-42 11721 similarly hot (but prohibitively distant). The three AME-hosts will thus be strong ultra-violet sources, the wavelength-regime in which excitation bands for nanodiamonds lie $^{21,22}$. For example, in the $\sim 200 \mathrm{~nm}$ band $^{22}$, model stellar-surface fluxes ${ }^{27}$ strongly depend on temperature: boosting $T_{\text {eff }}$ by $10 \%$ doubles this flux. Hence a temperature threshold may prohibit detecting IR features of nano-diamonds around slightly cooler stars. Applying a cut-off of $T_{\text {eff }} \approx 10,000 \mathrm{~K}$ for excitation, and then predicting microwave fluxes on the basis of source distance and the observed range of $\mathrm{L}(\mathrm{AME}) / \mathrm{L} *$, we find no other systems in our sample that should clearly show IR-features plus AME. As excitation also occurs ${ }^{21}$ near the ionization limit of $\sim 8 \mathrm{eV}$, if charged nano-diamonds are AME carriers, there may also be a link to detections of AME in environments with ionised gas $^{28}$.

Other AME carrier-particles have been proposed ${ }^{4,18,19}$, but discs are the new environment where the candidates have been spatially located. We do not rule out that other hydrocarbons can also

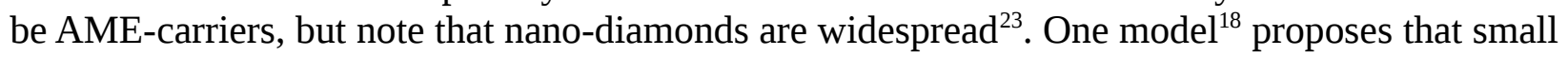
AME-carriers of only 8-15 C-atoms could also reproduce diffuse interstellar bands (DIBs), and the smallest nano-diamond form (adamantane, $\mathrm{C}_{10} \mathrm{H}_{16}$ ) is in this regime. Further, the fullerene ion $\mathrm{C}_{60}{ }^{+}$is the only identified ${ }^{30}$ DIB carrier, and carbon 'onion' structures such as $\mathrm{C}_{60}$ have been proposed as sites for nano-diamond formation ${ }^{9}$. $\mathrm{C}_{60}$ is now known in one HAe disc, and in two evolved-star envelopes hosting nano-diamonds ${ }^{29}$. Hence, if fullerenes and similar species are rather ubiquitous and can provide viable production-sites, this may be a route to generating nanodiamonds of sizes $~ 10-700$ C-atoms that could explain AME from diffuse interstellar gas and from dense circumstellar discs.

Solar system nano-diamonds may have been made in the proto-solar disc and/or inherited from previous generations of evolved stars. In star-systems generally, disc evaporation and stellar winds could expel nano-diamonds back into the interstellar medium. This provides a testable hypothesis, where the $3.47 \mu \mathrm{m}$ nano-diamond absorption features in dense interstellar clouds ${ }^{10,23}$ may correlate with AME sight-lines. Given a widespread distribution, nano-diamonds could present an alternate solution for the problem of poor correlation of AME and PAH distributions ${ }^{4}$.

\section{References:}

1. Kogut, A. et al. Microwave Emission at High Galactic Latitudes in the Four-Year DMR Sky Maps. Astrophys. J. Lett. 464, L5-L9 (1996).

2. Draine, B. T. \& Lazarian A. Electric Dipole Radiation from Spinning Dust Grains. Astrophys. J. 508, 157-179 (1998).

3. Planck consortium et al., Planck intermediate results. XXIII. Galactic plane emission components derived from Planck with ancillary data. Astron. Astrophys. 580, id.A13 (2015).

4. Hensley, B. S., Draine, B. T. \& Meisner, A. M. A case against spinning PAHs as the source of the anomalous microwave emission. Astrophys. J. 827, id.45 (2016).

5. Acke, B. \& van den Ancker, M. E. A survey for nanodiamond features in the 3 micron spectra of Herbig Ae/Be stars. Astron. Astrophys. 457, 171-181 (2006).

6. Keller, L. D. et al. PAH Emission from Herbig Ae/Be Stars. Astrophys. J. 684, 411-429 (2008). 
7. Goto, M. et al. Spatially Resolved $3 \mu \mathrm{m}$ Spectroscopy of Elias 1: Origin of Diamonds in Protoplanetary Disks. Astrophys. J. 693, 610-616 (2009).

8. Rafikov, R. R. Microwave Emission from Spinning Dust in Circumstellar Disks. Astrophys. J. 646, 288-296 (2006).

9. Ott, H. Nanodiamonds in meteorites: properties and astrophysical context. J. Achiev. Mat. \& Manufac. Eng. 37, 779-784 (2009).

10. Allamandola, L. J., Sandford, S. A., Tielens, A. G. G. M. \& Herbst, T. M. Infrared spectroscopy of dense clouds in the C-H stretch region - Methanol and 'diamonds'. Astrophys. J. 399, 134-146 (1992).

11. Mohanty, S. et al. Protoplanetary Disk Masses from Stars to Brown Dwarfs. Astrophys. J. 773, id.168 (2013).

12. Menu, J. et al. The structure of disks around intermediate-mass young stars from midinfrared interferometry. Evidence for a population of group II disks with gaps. Astron. Astrophys. 581, id.A107 (2015).

13. Blades, J. C. \& Whittet, D. C. B. Observations of unidentified infrared features in the premain sequence star HD 97048. Mon. Not. Royal Astron. Soc. 191, 701-709 (1980).

14. Guillois, O., Ledoux, G. \& Reynaud, C. Diamond Infrared Emission Bands in Circumstellar Media. Astrophys. J. 521, L133-L136 (1999).

15. Miyahara M. et al. Unique large diamonds in a ureilite from Almahata Sitta $2008 \mathrm{TC}_{3}$ asteroid. Geochim. Cosmochim. Acta 163, 14-26 (2015).

16. Acke, B. \& van den Ancker, M. E. ISO spectroscopy of disks around Herbig Ae/Be stars. Astron. Astrophys. 426, 151-170 (2004).

17. Geers, V. C. et al. C2D Spitzer-IRS spectra of disks around T Tauri stars. II. PAH emission features. Astron. Astrophys. 459, 545-556 (2006).

18. Bernstein, L. S., Clark, F. O., Cline, J. A. \& Lynch, D. K. The Diffuse Interstellar Bands and Anomalous Microwave Emission May Originate from the Same Carriers. Astrophys. J. 813, id.122 (2015).

19. Hensley, B. S. \& Draine, B. T. Modeling the Anomalous Microwave Emission with Spinning Nanoparticles: No PAHs Required. Astrophys J. 836, id.179 (2017).

20. Pascucci, I. et al., Low Extreme-ultraviolet Luminosities Impinging on Protoplanetary Disks. Astrophys. J. 715, id.1 (2014).

21. Bauschlicher, C. W., Liu, Y., Ricca, A., Mattioda, A. L. \& Allamandola, L. J. Electronic and Vibrational Spectroscopy of Diamondoids and the Interstellar Infrared Bands between 3.35 and $3.55 \mu \mathrm{m}$. Astrophys. J. 671, 458-469 (2007).

22. Steglich, M., Huisken, F., Dahl, J. E., Carlson, R. M. K. \& Henning, T. Electronic Spectroscopy of FUV-irradiated Diamondoids: A Combined Experimental and Theoretical Study. Astrophys. J. 729, id.91 (2011).

23. Pirali, O. et al., Infrared Spectroscopy of Diamondoid Molecules: New Insights into the Presence of Nanodiamonds in the Interstellar Medium. Astrophys. J. 661, 919-925 (2007).

24. Terada, H., Imanishi, M., Goto, M. \& Maihara, T. Detection of the unusual $3.5 \mathrm{mu}$ m feature in the Herbig Be star MWC 297. Astron. Astrophys. 377, 994-998 (2001).

25. Habart, E., Testi, L., Natta, A. \& Carbillet, M. Diamonds in HD 97048: A Closer Look. Astrophys. J. 614, L129-L132 (2004). 
26. Siebenmorgen, R., Kruegel, E. \& Mathis, J. S. Radiative transfer for transiently heated particles. Astron. Astrophys. 266, 501-512 (1992).

27. Castelli, F. \& Kurucz, R. “The Grids of ATLAS9-ODFNEW models and fluxes”, available at http://www.stsci.edu/hst/observatory/crds/castelli_kurucz_atlas.html (2004).

28. Scaife A. M. M. et al., Microwave observations of spinning dust emission in NGC6946. Mon. Not. Roy. Astron. Soc. 406, L45-L49 (2010).

29. Roberts, K. R. G., Smith, K. T. \& Sarre, P. J. Detection of C60 in embedded young stellar objects, a Herbig Ae/Be star and an unusual post-asymptotic giant branch star. Mon. Not. Royal Astron. Soc. 421, 3277-3285 (2012).

30. Campbell, E. K., Gerlich, D. \& Maier J. P, Laboratory confirmation of C60+ as the carrier of two diffuse interstellar bands. Nature 523, 322-323 (2015).

Acknowledgments: The NRAO is a facility of the National Science Foundation operated under cooperative agreement by Associated Universities, Inc. Infrared spectra are presented from the processed data archives of ESA's Infrared Space Observatory and NASA's Spitzer Space Telescope. A. Avison at JBCA reduced the ALMA observations of HD 97048. AMMS gratefully acknowledges support from the European Research Council under grant ERC-2012-StG-307215 LODESTONE. We thank the staff of the Lord's Bridge Observatory for their assistance in the operation of the AMI. The AMI is supported by the University of Cambridge and the STFC.

Author contributions: JSG led the project, analysed GBT and ISO data, coded initial models, and drafted the paper. AMMS analysed ATCA data, contributed AME and coding expertise, and wrote modeling sections of the paper. DTF, DAG, BSM and AMSS contributed instrument, observation and software support and commented on the paper.

\section{Additional information}

Supplementary information is available for this paper. Correspondence and requests for materials should be addressed to JSG.

\section{Competing interests}

The authors declare no competing financial interests.

Figure 1. Plots show the profile of the anomalous microwave emission and the modelled probabilites for nano-diamond size and abundance. (Left panel:) Data points for V892 Tau, with dust and wind model subtracted to leave the AME residual. Error bars include the range of allowed subtracted wind+dust signals, plus flux uncertainties (or a minimum of $10 \%$ calibration uncertainty), added in quadrature. Upper limits are $+2 \sigma$ (gaussian statistics are used throughout). Dashed line shows the Maximum Likelihood (ML) fit; the solid line shows the model using the parameters' expectation values; thin lines are 24 samples randomly drawn from the posterior distribution. (Right panel:) Parameter space for model variables a (nano-diamond radius) and nano-diamond:carbon abundance, showing marginalised posterior probability. Solid lines mark positions of ML parameter values; dashed lines are 16, 50, 84\% quantiles on the 1D posteriors (equivalent to $-1 \sigma$, mean, $+1 \sigma$ ); contours show $68,95,99 \%$ levels on the 2D posterior.

Figure 2. AME data points and models for HD 97048; details as in Figure 1.

Figure 3. AME data points and models for MWC 297; details as in Figure 1. 


\section{Methods}

\section{$\underline{\text { Radio Observations }}$}

The Australia Telescope Compact Array was used to observe 9 discs of HAe stars. The array was in the H214 hybrid configuration, with baselines in the range from 92 to 4383 metres between the six 22m antennas. Signals from the $\mathrm{C} / \mathrm{X}$ and $\mathrm{mm}$ receivers were correlated using the Compact Array Broadband Backend. The survey was designed to search for AME, and observations used bands at 5.5, 8.8, 18, 24, 32, 38, 91 and $97 \mathrm{GHz}$, with bandwidths of $2048 \mathrm{MHz}$. In total, 28 datasets were obtained for the nine sources over 6-11 October 2010. For MWC 297, observations were made in all 8 frequency bands, each lasting from 6 to 130 minutes. Only four frequency bands were observed for HD 97048, for durations of 20-160 minutes. Multiple scans were made in all cases (except the two lowest frequencies observed for MWC 297). Uranus was used as the primary flux calibrator along with secondary calibrators (1934-638 for MWC 297 and 0537-441 for HD 97048; the latter is slightly variable in flux, so pairs of data points are shown for primary and secondary calibration). The data are publicly available under project code C2426. Archival data for HD 97048 were also processed to provide additional frequencies (from project code C1794, using a range of ATCA configurations).

Observations of V892 Tau were made at the 100m Green Bank Telescope in West Virginia,. Thirty HAe and CTTS systems were observed in a flux-limited survey covering discs with 1.3 mm flux $>90$ mJy in Taurus and Ophiuchus. The Ka-band receiver was used with the Caltech Continuum Backend; the CCB uses optimized detector circuits and $4 \mathrm{kHz}$ beam-switching to suppress instrumental gain fluctuations. Four frequency channels are obtained at 26-29.5, 29.533.0, 33.0-36.5 and 36.5-40 GHz. Photometry utilised an on-the-fly nod, with four 10-second phases in a 70-second observation. Seven repeats of this sequence were made for V892 Tau on 21 April 2007, immediately after a skydip and calibration check, and before an observation of DL Tau. The latter showed a normal power-law spectrum across the 4 sub-bands with an index of 1.7 (in the convention of positive index for rising flux at higher frequencies), with correlation coefficient of $r=0.99$. Flux densities were established by observing the primary calibrators 3C 48 and 3C 147, which have power-law spectra (measured indices of -1.18 and -1.09 respectively). Neither calibrator was undergoing flux changes at similar frequencies at the time ${ }^{31}$. The individual data points for V892 Tau are shown in Fig. S1. The archived data are available under project code AGBT07A-038.

V892 Tau was also observed with AMI-LA, the Large Array of the Arcminute Microkelvin Imager $^{32}$. This comprises eight $13 \mathrm{~m}$ antennas sited at the Mullard Radio Astronomy Observatory at Lord's Bridge, Cambridge, UK. The telescope observes in the band 13.5-17.9 GHz with eight $0.75 \mathrm{GHz}$ bandwidth channels, but the two lowest frequency channels have lower response in this frequency range and suffer from interference; the effective frequency here was $16.1 \mathrm{GHz}$. In total ten HAe/CTTS sources were observed in this band from July and September 2011. AMI-LA flux calibration is performed using observations of 3C286, 3C48 and 3C147, with I+Q flux densities for these sources in the AMI-LA channels consistent with the updated VLA calibration scale; polarization and airmass are also corrected for. Tests show fluxes are accurate to $\leq 5 \%$.

MWC 297 was also observed with the GBT W-band receiver, on 26 September 2016 (project code AGBT16B-390). Fast scans of the telescope's 10-arcsecond beam were made to extract the source signal from the background level. The effective on-source time per pass was $\sim 5$ seconds, with 64 scans made in total. Sky conditions were good at $72 \mathrm{GHz}$, with zenith opacity of 0.26 , 
but the beam size varied with telescope temperature; the source is point-like within this limitation (Fig. S2). The signal-to-noise ratio is 14, and the $72 \mathrm{GHz}$ flux is $85 \pm 11 \mathrm{mJy}$ (for an error budget of $8 \%$ noise and $10 \%$ in calibration); the flux calibrator was $1751+0939$.

All new results for the AME sources are presented in Table S1. Literature flux densities ${ }^{20,33-40}$ from VLA, ATCA, CARMA, BIMA and ALMA were included in our analysis for consistency checks, to fill in frequency coverage, and to fit combined signals from wind plus dust (Fig. S3). We independently reduced an archival dataset ${ }^{39}$ of HD 97048 from the Atacama Large Millimetre Array to generate a flux error at $106 \mathrm{GHz}$. For HD 97048 (CU Cha), the archival ATCA data (Table S1) were recently published ${ }^{40}$. Our results agree within the errors, but are systematically slightly higher in flux, probably due to our use of phase self-calibration of the field.

\section{Radio Data Analysis}

The total radio-frequency range in the analysis spans 1.4-115 GHz. The fluxes of dust and wind components in each system were fitted and subtracted to yield a minimum AME residual signal. The winds were characterised at frequencies below $\sim 10 \mathrm{GHz}$, and temporal variability was included where possible (Fig. S3). The wind indices resulting from the fits are all within known bounds, which extend from -0.1 up to 0.6 in simple optically-thin geometries, increasing to 2 in optically-thick cases. Dust signals were fitted at the high frequency end, with maximised numbers of frequencies set to have no AME. In addition, dust spectral indices were confined to the range 2-4, appropriate for large to small grain sizes, emitting as blackbodies and inefficient greybodies respectively. An overall fitting requirement was that residuals after dust-plus-wind subtraction should not be negative, within errors. Although SR 21 is a weak AME candidate, this source is a pair, with the low $34 \mathrm{GHz}$ flux ${ }^{20}$ including only the A-component. Hence the GBT flux at $31.25 \mathrm{GHz}$ (detected at only 3.5б), suggesting a small candidate residual, appears to be mainly from the less-studied B-component (which has no independent IR spectroscopy).

The highest frequency for each source was also used to estimate masses of dust. The 106-115 $\mathrm{GHz}$ fluxes were scaled by source distance, and then converted to mass via a V892 Tau model ${ }^{33}$. Thus masses for HD 97048 and MWC 297 ignore any variations between host stars and disc geometries, but benefit from using the most optically-thin (longest) wavelength. Literature fluxes at 230-345 GHz lie below extrapolations to our dust fits by factors of $2 \pm 0.5$, indicating similar optical depths in each case, and so reasonably robust results from the scaling approach.

\section{Infrared Spectra}

The heritage archive of the Infrared Space Observatory (ISO) yielded nano-diamond spectra of V892 Tau, MWC 298 and HD 97048 (Fig. S4). The 3.43, $3.53 \mu \mathrm{m}$ features lie in the '1D' subband of the Short-Wave Spectrometer, and highly-processed data products ${ }^{41}$, HDDP, are shown, where artificial fringing has been reduced. These spectra are discussed in the literature ${ }^{42}$ and also have ground-based equivalents ${ }^{5}$. Fig. S5 shows additional archival spectra, from ISO and the Spitzer Space Telescope, to demonstrate PAH features around $6.2 \mu \mathrm{m}$ observed for our sample of HAe stars. Another nano-diamond peak is predicted ${ }^{23}$ at $\sim 6.88 \mu \mathrm{m}$ and its presence was suggested $^{42}$ for HD 97048, but in discs it can be blended with a $6.85 \mu \mathrm{m}$ line of water vapour.

Table S3 lists fluxes and limits for the $3.53 \mu \mathrm{m}$ nano-diamond feature for all the sources in our sample. The values listed are mainly from a survey ${ }^{16}$ made with ISO; these agree only within $\sim 60 \%$ with ground-based values ${ }^{5}$ due to difficulties in absolute calibration, and so the errors were set conservatively at a $5 \sigma$ level $^{16}$. For T Tau, we estimated a limit from an archival ISO HDDP, 
and for SR 21 and IC 2087 IR we used the rms in ground-based spectral observations ${ }^{45,46}$ to generate a similar error. Fig. S6 plots the AME detections and limits against the fluxes for the $3.53 \mu \mathrm{m}$ nano-diamond and $6.2 \mu \mathrm{m}$ PAH features (Table S3).

\section{$\underline{\text { Statistics }}$}

The probability $\mathrm{P}_{\text {nano }}$ was corrected for biases. ATCA targets were chosen to have bright PAHs, and e.g. the faintest $6.2 \mu \mathrm{m}$ line in this sample has a flux of $610^{-15} \mathrm{~W} / \mathrm{m}^{2}$; $20-40 \%$ of the HAe stars in the ISO nano-diamond survey ${ }^{16}$ fall below this. GBT targets were chosen to have $1.3 \mathrm{~mm}$ dust flux $>90 \mathrm{mJy} ; 50 \%$ of the $I S O$ survey fall below this, e.g. using a mean spectral index $\sim 2.5$ to extrapolate $0.85 \mathrm{~mm}^{\text {fluxes }}{ }^{48}$. Weighting by the 9 ATCA and 5 GBT targets, the proportion of HAes that were searched for nano-diamonds but not for AME is $\sim 30-45 \%$, so $\mathrm{P}_{\text {nano }}$ is de-biased from 0.037 to $\sim 0.06$. The probability of getting 3 nano-diamond hosts in a random sample of 14 HAe stars is then $\mathrm{P}=\mathrm{P}_{\text {nano }}{ }^{3}\left(1-\mathrm{P}_{\text {nano }}\right)^{11} \mathrm{C}_{14,3}$ where $\mathrm{C}_{n . \mathrm{k}}=\mathrm{n} ! /(\mathrm{n}-\mathrm{k}) ! \mathrm{k}$ ! is the number of ways of drawing 3 stars out of 14 . This is then divided by $\mathrm{C}_{14,3}$, the number of ways of picking 3 AMEhosts out of 14 radio targets, to yield $\mathrm{P}_{\text {chance }}$ for the $3 \mathrm{AME}$ hosts to also have nano-diamonds. One multiple-source (Fig. 3e below) could be biased against AME detection, but omitting this object would only increase false-alarm probabilities by a factor $1 /\left(1-\mathrm{P}_{\text {nano }}\right), \approx 1.04-1.06$.

\section{AME Modelling}

We fit for the AME carrier-particle parameters using two burn-in periods of 1000 steps each, followed by a production run, of the EMCEE EnsembleSampler, using 12 chains each of 10000 steps. We calculate the autocorrelation length, $\tau$, using the ACOR package and discard the first 100t samples before calculating the $16,50 \& 84 \%$ quantiles from the $1 \mathrm{D}$ posteriors to extract the expectation value of each parameter and its one sigma bounds. We use the raw likelihoods within the prior volume to extract the maximum likelihood parameter values. Each plot in Figures 1-3 shows maximum likelihood and expectation fits to the AME spectrum, along with 24 sample fits randomly drawn from the posterior. The parameter plots show the probability distributions of nano-diamond abundance and radius, and representative uncertainties are also listed in Table 1.

Data Availability

The processed data that support the plots within this paper and other findings of this study are available from the corresponding author upon reasonable request. The raw data from ATCA and GBT are available from the respective telescope archives, under the project codes listed above.

\section{Additional References}

31. Perley, R. A. \& Butler, B. J. An Accurate Flux Density Scale from 1 to 50 GHz. Astron. J. 204, id.19 (2013).

32. Zwart, J. T. L. et al., The Arcminute Microkelvin Imager. Mon. Not. Roy. Astron. Soc. 391, 1545-1558 (2008).

33. Hamidouche, M. Aperture Synthesis Imaging of V892 Tau and PV Cep: Disk Evolution. Astrophys. J. 722, 204-211 (2010).

34. Phillips, N. M. Far-infrared and sub-millimetre surveys of circumstellar discs. PhD thesis, University of Edinburgh, https://www.era.lib.ed.ac.uk/handle/1842/5032 (2011).

35. Dzib, S. A. et al., The Gould's Belt Very Large Array Survey. IV. The Taurus-Auriga Complex. Astrophys J. 801, id.91 (2015). 
36. di Francesco, J. et al., Millimeter and Radio Interferometry of Herbig Ae/Be Stars. Astrophys. J. 482, 433-441 (1997).

37. Skinner, S. L., Brown, A. \& Stewart, R. T. A high-sensitivity survey of radio continuum emission from Herbig Ae/Be stars. Astrophys. J. Supp. 87, 217-265 (1993)

38. Henning, T., Pfau, W., Zinnecker, H. \& Prusti, T. A 1.3-mm Survey of Circumstellar Dust around Young Chamaeleon Objects. Astron. Astrophys. 276, 129-141 (1993).

39. Dunham, M. et al., An ALMA Search for Substructure, Fragmentation, and Hidden Protostars in Starless Cores in Chamaeleon I. Astrophys. J. 823, id.160 (2016).

40. van der Plas, G. et al., A cavity and further radial substructures in the disk around HD 97048. Astron. Astrophys. 597, id.A32 (2017).

41. Frieswijk, W., Shipman, R.F., Lahuis, F. \& Hormuth, F. "SWS AOT-1 High Resolution Processing Documentation” (technical report number 52, European Space Agency, available at http://ida.esac.esa.int:8080/hpdp/technical reports/technote52.pdf, 2007).

42. Van Kerckhoven, C., Tielens, A. G. G. M. \& Waelkens, . Nanodiamonds around HD 97048 and Elias 1. Astron Astrophys. 384, 568-584 (2002).

43. Wright, C. M. et al., Resolving structure of the disk around HD100546 at $7 \mathrm{~mm}$ with ATCA. Mon. Not. Roy. Astron. Soc. 453, 414-438 (2015).

44. Mason, B. S. et al., A $31 \mathrm{GHz}$ survey of low-frequency selected radio sources. Astrophys. J. 704, 1433-1447 (2009).

45. Tanaka, M., Sato, S., Nagata, T. \& Yamamoto, T. Three micron ice-band features in the Rho Ophiuchi sources. Astrophys. J. 352, 724-730 (1990).

46. Brooke, T. Y., Sellgren, K. \& Smith, R. G. A Study of Absorption Features in the 3 Micron Spectra of Molecular Cloud Sources with H2O Ice Bands. Astrophys. J. 459, 209-215 (1996).

47. Fairlamb, J. R.,Oudmaijer, R. D., Mendigutía, I., Ilee, J. D. \& van den Ancker, M. E. A spectroscopic survey of Herbig Ae/Be stars with X-shooter - I. Stellar parameters and accretion rates. Mon. Not. Royal Astron. Soc. 453, 976-1001 (2015).

48. Sandell, G., Weintraub, D. A. \& Hamidouche, M. A Submillimeter Mapping Survey of Herbig AeBe Stars. Astrophys. J. 727, id.26 (2011). 